\title{
Superstitious Behaviour in Sportspersons in Relation to Their Performance at Intercollegiate Level
}

\author{
Bhoomi Gupta ${ }^{1}$, Siddharth Dutt ${ }^{2}$ \\ ${ }^{1}$ M.Sc. Counselling Psychology, Department of Psychology, Christ University, Bangalore \\ ${ }^{2}$ Assistant Professor, Department of Psychology, Christ University, Bangalore \\ Corresponding author: Siddharth Dutt \\ Email: siddharth1024@gmail.com
}

\begin{abstract}
Background: Superstitions are beliefs that fate is governed by uncontrollable external forces. Superstitions play a key role in sports since they associate with activities involving danger and lack of predictability. There has been a dearth of studies regarding the effect such superstitious beliefs have on athletes' performance, and on the looking superstitions from a qualitative perspective, specifically on Indian population, hence this study aims to explore the various superstitious beliefs (SB) endorsed by team sportspersons at intercollegiate level and analyze how these beliefs affect their sports performance. This study also aimed at analysing the gender differences within different team sports.

Methods: Data was collected using purposive and snowball sampling technique. 50 sportspersons from badminton, basketball, football, cricket, hockey, and volleyball participated. Semi- structured interviews were conducted to obtain rich data. Content analysis was carried out to obtain the emerging themes.

Results: The major themes which emerged from the data include nature, origin, frequency, impact, intentionality, evolution, and orientation of superstitions in reality.

Conclusion: Further research is needed in the Indian context regarding sensitivity towards superstitious and the need to incorporate them in coaching for enhancing performance.
\end{abstract}

Keywords: superstitions, superstitious behavior, team sports, athletes, performance.

(Paper received $-14^{\text {th }}$ May 2019, Peer review completed $-24^{\text {th }}$ June 2018)

(Accepted - 28 $8^{\text {th }}$ June 2019)

\section{INTRODUCTION}

Superstitions are considered to be natural psychological mechanisms which help the athlete to cope with the stress associated with the competitive situation and to perform at their best under pressure. Since, superstitions are associated with activities which involve danger and lack of predictability, hence they play a key role in sports. Since superstitious rituals are universal, and common across various cultures because they aid in attaining better performance, and there have been a dearth of studies regarding the effect such superstitious beliefs can have on performance of athletes, specifically on Indian population, hence this study seeks to qualitatively explore the various superstitious beliefs (SB) which are practised by team sportspersons at intercollegiate level and analyse how these superstitious beliefs affect their sport performance. This study also seeks to analyse the gender differences within different team sports associated with these superstitions. Superstitions arise when an athlete performs, either positively or negatively and then tries to establish a "cause and effect" relationship by reviewing these behaviours at the end of the day. Studies have observed that superstitions are innate psychological mechanisms which help the athlete to cope with the stress associated with the competitive situation and to perform their best under stress. According to Maller and Lundeen [1], in major stressful situations, one starts to believe that their fate is in the hands of unknown 
external powers over which they have no control. According to the study conducted by Brevers and others [2], it was found that commitment to rituals increases when the uncertainty and importance of the game are greater. Schippers and Van Lange [3] found that elite athletes are more prone to use superstitious rituals in high uncertainty and anxiety-provoking conditions and significant competitions. According to the study conducted by Hamerman and Morewedge [4] , it was found that preference and assignment to a lucky (vs. unlucky) item lead to greater confidence of achieving performance goals but not learning goals. Also, increase in the uncertainity of goal acheievement lead to an increase in superstious behaviours. Another study by Chinchanachokchai and others [5] asserted that passive superstitious beliefs (low illusion of control) increase the likelihood of engaging in risky behaviors. Additionally, anxiety relates to the employment of rituals [3].

Pre-Performance Rituals have been shown to help performers better focus their thoughts on task-relevant cues, overcome natural instincts to dwell on negatives thoughts, reduce the impact of distractions during performance. No significant difference was seen in performance when engaging in Pre-performance rituals and superstitions, when the athletes' familiarity with the sport and practise increases. One of the participant in the study conducted by Cherrington [6] reported packing his bag, listening to hip hop music and choosing the kit to be worn during the game as a pre-game ritual, which has been developed and strengthened over time. In such situations, these superstitions, for example lucky charms, provide them the assurance that they have given their best in the game. According to the study conducted by Tahir and others [7], it was reported that superstitions originated from the Hindu culture of sub-continent and got transmitted from generation to generation. It was also concluded that superstitious people aren't able to take any decision without involvement in such beliefs. According to the study conducted by Chen and Young [8], it was found that belief in stable luck seemed to positively associate with the inclination towards superstition among people with Asian cultural background. It can also be argued that the longer and greater an athlete's involvement in a sport, the stronger are the superstitions. This can be substantiated by the study conducted by Neil and others [9] that higher competitive level and greater involvement in a sport increases the prevalence of superstitions.

Low skill athletes in an easy condition and high skill athletes in difficult condition tend to score higher in superstitious behaviour [10]. Raalte and others [11] suggested that highly superstitious athletes tend to attribute unsuccessful results to others, in order to protect their self-worth. Superstitions get widely reflected in our language and within a myriad of daily routine rituals, i.e. common gestures and expressions like saying good luck or crossing fingers etc. There have been variations in superstition varying from sport to sport, and the roles assumed within a sport too. The most significant and extensive study reported till date on superstition in sport is the study conducted by Gregory [12]. Analysis revealed that team sport athletes provide greater evidence for superstitions relating to equipment and its use; order of entering the arena or their playing position; dressing room activities; repetitive rituals; and sports personalities. The most obvious variations in sport superstitions is in the nature of sport. Gregory and Petrie's study [13], observed superstitions relating to uniform, equipment and clothes. According to Becker [14], the most customary superstition involved wearing a particular article of lucky clothing.

Gregory and Petrie [13] also found evidences of superstitions in their review of literature among jockey players, curler players, volleyball and basketball players, race car drivers, boxers and swimmers. Samuelsen [15] provides an array of examples of superstitious beliefs in football, basketball, golf, boxing, harness racing, baseball, tennis, bowling and diving. According to Coffin [16], there are three types of sport superstitions, on the basis of their origin - those beliefs and practices which they bring with them from their main culture; the long-standing superstitions associated with their sport; their personal superstitions or eccentricities. Gregory and Petrie [13] distinguished sport superstitions from general superstitions, on the basis of the nature of superstition, under the sub-categories of equipment, uniform, clothes, routine, spectators, religion, numbers, hair, hands, charms, coins and food. Becker [14] had perhaps made the most collaborative attempt to categorize sport superstitions or at least locate them in logical groups which include (1) clothing, (2) manner of dressing, (3) numbers, (4) practices to prevent or cure injury, (5) equipment care and use, (6) 
practices before and during contests, and (7) omens, premonitions and beliefs about the result of a contest. Borrowed articles, new clothing was seen to disrupt an athlete's performance.

The most obvious variations in sport superstitions which is expected is in the nature of sport. In Gregory and Petrie's [17] study of intercollegiate basketball, volleyball, ice hockey, track and field, swimming and tennis players, it appeared that many superstitions were associated to the nature of the particular sport concerned. Whereas, Womack [18] suggested a great deal of consistency from sport to sport. In discussing day-of-the-game rituals, she noticed eating habits, precise ordering of the route to the game as described by baseball, football, basketball and ice hockey players. Basketball players also believe in certain rituals such as the last person to shoot a basket during the warm-up is found to have a good game. Wiping the soles of sneakers and bouncing the ball before taking a foul shot is believed to bring good luck. A study by Chris [19] found that elite basketball players made more foul shots when they performed their preferred pre-routines than when they deviated from the behavioural pattern. Football players believe in certain rituals such as double numbers on a player's uniform brings good luck. And it's bad luck for a professional football player to take a new number when he is traded to another team. A mascot is an important good luck symbol. It is a well-known fact that many of Indian cricketers are known to swear by certain personally practiced superstitions which they use to achieve success. For example Virat Kohli is known to be obsessed with a pair of lucky gloves; Yuvraj Singh was loyal towards the number 12, Zaheer Khan was penchant to carry a yellow handkerchief along, and Sachin Tendulkar chose to wear his left batting pad before putting the right one. Even Nita Ambani who owns Mumbai Indian cricket team was found to repetitively chant over a picture which she places in her bag when her team used to perform. Former Indian batsman and current coach of the Zimbabwe national team, Lalchand Rajput mentioned that some players stand while slipping on batting pads since it's believed that sitting brings bad luck. Cricketer Siddhesh Lad believes that watching movies a day before the match or using social media on the day of the match is associated with bad luck. Observing the variations in endorsements of types of superstitions among the intercollegiate athlete subjects of Gregory and Petrie's study [13], it was observed that superstitions relating to uniform, equipment and clothes were most often cited. Becker [14] also indicated that the most customary superstition involved wearing a particular article of lucky clothing. Family, friends, school, the mass media and personal experiences are known to be some of the most important sources in the transmission of such beliefs [17]. There have been also some evidences of sex differences. Gregory and Petrie [13] found that women college students continued to endorse more superstitious beliefs related to personal appearance including uniform, hair, specific hair accessories, team cheers, hands and social pre-game-night activities which stem from general superstitions as compared to men. The male athletes listed more superstitions relating indirectly to business practices, equipment, religious practices and repetitive actions than did the female athletes. Another study conducted by Neil and others [9] reported that men tend to be more superstitious and report more sport related superstitions. This finding can also be supported by another study conducted by Conklin [20] in which it was reported that men reported superstitions relating to sports, business related activities. Another study [7] stated that women subscribe more to superstitious beliefs than men, and rural people are more superstitious than urban people. Also, people with low education status tend to be more superstitious as compared to highly educated people, and the people who have high levels of family tensions tend to be more superstitious than those of having low level of family tensions.

According to the prior researches, the only means of obtaining data about superstitions from athletes is using direct observation and interviewing. Since superstitious rituals are universal, constant and common across various cultures because they aid in attaining better performance, and there have been a dearth of studies regarding the effect such superstitious beliefs can have on performance of athletes, specifically on Indian population, hence this study seeks to qualitatively explore the various superstitious beliefs (SB) which are practised by team sportspersons at intercollegiate level and analyse how these superstitious beliefs affect their sport performance. This study also seeks to analyse the gender differences within different team sports associated with these superstitions. 


\section{METHODOLOGY}

The aim of the present study was to explore how superstitious beliefs of sportspersons at intercollegiate level affect their sports performance. The sample size was 50. The location of the study was Bangalore and Delhi. The participants, aged 18-25, were approached from Christ University, Bangalore and academies in Delhi. The various sports included for the study were football, basketball, cricket and volleyball, hockey, netball, table tennis (doubles), throw ball, water-polo, badminton (doubles). The sample consisted both of male and female sportspersons. Participants were approached using purposive and snowball sampling technique in order to obtain rich data relevant to the study. A pilot study was conducted with 2 participants to check the timing, administration and efficacy of the semi-structured interview schedule. The main phase was then carried out with 50 participants from badminton, football, basketball, cricket, volleyball etc. Informed consent was taken from the participants, where they were assured of their right to withdraw from the study at any given point of time. The interview schedule carried 10 questions and the duration of the interview was 20-45 minutes. The various tools used in the current study included demographic form; consent form and semi-structured interview schedule. The interview guide was validated using researcher triangulation by three experts from the field of sport psychology. Verbatim was taken from the participants. The data was exported to an excel sheet in order to provide structure to the data. The emerging themes and the similar contents was clubbed together and colour coded in order to analyse the data effectively and to generate the possible nature, persistence, origin, frequency, impact, intentionality, orientation to reality, and changes in the superstitious beliefs and practices. The inclusion criterion was literate male and female intercollegiate sportspersons from team sports, aged 18 to 25 from colleges and sports academies from Bangalore and Delhi. The exclusion criterion for the study included the athletes who are currently involved in sports, with no particular cultural or religious background.

\section{RESULTS}

The data was analysed using Content Analysis to reduce large amount of unstructured content, highlight important aspects of the content, and examine the trends and relationships emerging in the text and also to gain insights into it. Table I describes the socio-demographic details of the participants including their education level, sport, gender and state they belong to.

The following figure (figure 1.0) illustrates the frequency of participants to indulge in superstitious practices. The figure suggests that 39 participants $(78 \%)$ indulge in superstitious activities, whereas 11 participants didn't believe in superstitions and didn't use them in their sport.

\section{Meaning of Superstitions}

The analysis looked at what the participants meant by superstitions, both in sports and in daily life. 20 participants responded that superstitions are strong beliefs, habits, rituals which guides ones actions and can be based on luck. This was substantiated by:

"These are quick decisions you take when you play- who to pass, when to shoot, who to block etc." (Participant 11)

The analysis looked at what the participants meant by superstitions, both in sports and in daily life. 20 participants responded that superstitions are strong beliefs, habits, rituals which guides ones actions and can be based on luck. This was substantiated by:

"These are quick decisions you take when you play-who to pass, when to shoot, who to block etc." (Participant 11)

"Superstitions are something which gives positive energy" (Participant 42)

"Superstitions are beliefs, rituals which makes things go our way." (Participant 24)

23 participants responded that superstitions are false, illogical beliefs which people blindly believe which they think that impacts their routine activities. This was substantiated by:

"Negative false belief which people choose to believe" (Participant 15)

"Superstition is a blind belief which people think works." (Participant 28)

"Superstition is anything which distracts you or which is fashion, madness, passion." (Participant 40) 
Seven participants believed that these are strong beliefs, which may or may not depend on luck, makes them think that it is impacting their life and hence can be both logical and illogical, depending on their nature and purpose.

"Superstition is a false belief which supports what happens to you, makes good happen to you" (Participant 13)

"It is an unwanted belief which might boost confidence" (Participant 19)

Table 1: Socio- demographic details of the participants $(n=50)$

\begin{tabular}{|c|c|}
\hline Demographics & Number \\
\hline \multicolumn{2}{|l|}{ Age } \\
\hline $18-21$ & 31 \\
\hline $22-25$ & 19 \\
\hline \multicolumn{2}{|l|}{ Sport } \\
\hline Badminton & 1 \\
\hline Basketball & 18 \\
\hline Cricket & 6 \\
\hline Football & 15 \\
\hline Hockey & 3 \\
\hline Netball & 1 \\
\hline Table tennis & 3 \\
\hline Throw ball & 1 \\
\hline Volleyball & 1 \\
\hline Water polo & 1 \\
\hline \multicolumn{2}{|l|}{ Education } \\
\hline Pre-University & 7 \\
\hline Under-graduation & 28 \\
\hline Post-graduation & 15 \\
\hline \multicolumn{2}{|l|}{ Current State } \\
\hline Delhi & 12 \\
\hline Bangalore & 38 \\
\hline \multicolumn{2}{|l|}{ Gender } \\
\hline Female & 10 \\
\hline Male & 40 \\
\hline
\end{tabular}

\section{Nature of superstitions}

The analysis showed that four (8\%) participants reported that they used verbal superstitions such as reciting certain mantra, cheering up as a team, or praying (either silently or loudly as a team). This was substantiated by:

"We pray silently before starting the match". (Participant 19)

Further analysis revealed that 46 participants reported that they used non- verbal superstitions such as kit related, other gestures/ rituals and clothing related. Among these participants, six participants reported their superstitions as kit related, which can be substantiated by:

"I use the hologram side of the racket, and place my kit on the left side of the court so that I get enough space on the right side to play."(Participant 1);

"I have a particular bag which has my kit, which I always carry". (Participant 2)

Analysis showed that 36 participants reported that they used clothing related superstitions, which can be substantiated by:

"I wear 2 Nike ankle length socks in each leg, either white or black, which helps in improving my grip". (Participant 1); "I follow jersey number 15 for my matches." (Participant 5)

Analysis also revealed that eight participants reported that they either used a combination of superstitions. This can further be substantiated by:

"I touch the ground and then my eyes for respect." (Participant 14) 
"We as a team hold hands before the match and one person holds ball in the centre and prays." (Participant 14)

The following figure (figure 2.0) depicts the nature of superstitions which are performed by the participants. The figure depicts that four participants (8\%) performed verbal superstitions, whereas 46 participants (92\%) performed non-verbal superstitions.

\section{Origin of superstitions}

According to the analysis, five participants reported that their superstitions originated from their family. This can further be substantiated by:

"I started following this superstition \& my family validated it." (Participant 34)

16 participants reported that their superstitions originated from their own self. This was further substantiated by:

"I started following it because I saw that these helped improve my performance." (Participant 20)

29 participants reported that their superstitions originated from a combination of self, family and others.

"I developed the superstition by observing famous personalities like LeBron James, Michael Jordan; Kohli; Sachin Tendulkar" (Participant 17, 25)

"I tried reducing my belief in them but my coach told me not to experiment before matches, because of which I still follow it." (Participant 27)

"I personally developed this superstition in the beginning but then it got validated by my family and my coach." (Participant 36)

"I developed the superstition after being influenced by my teammates." (Participant 42)

\section{Frequency of performing superstitions}

Analysis indicated that 17 participants used these superstitions only during significant tournaments and matches. This can further be substantiated when the participants reported that:

"I use the superstition only when there are significant tournaments" (Participant 33)

Analysis also suggest that 33 participants used these superstitions both during their tournaments and practice sessions. This was substantiated by:

"I use it mostly during matches, but sometimes in practise sessions." (Participant 6)

The following figure (figure 3.0) depicts the frequency of superstitions which are performed by the participants. It can be observed that 33 participants (66\%) performed their superstitions both during tournaments and practise sessions, whereas 17 participants performed their superstitions only during tournaments.

\section{Impact of superstitions on performance}

Analysis suggested that 30 participants believed that their superstitions had an impact on only their psyche and didn't hold any reality basis. This can be supported by:

"Following the superstition improves my confidence and it constantly keeps nagging in my mind if I don't follow it. I follow it because it provides me better grip and comfort." (Participant 1)

Analysis also suggest that 18 participants believed that their superstitions hold significant basis in reality. This can be supported by:

"It helps increase my motivation, confidence and the respect towards sport increases. It also acts as a reinforcement." (Participant 14)

The following figure (figure 4.0) depicts the impact of superstitions on the performance of the participants. It can be observed that 30 participants $(60 \%)$ found their superstitions to have a logical basis, whereas 18 participants $(36 \%)$ believed their superstitions to be illogical. There were two participants (4\%) who believed that their superstitions are logical because they have an impact on their performance but also seem illogical because they aren't proved. 


\section{Purpose of superstitions}

Analysis also looked at the intentional nature of the superstitions. 27 participants believed that they performed their superstitions intentionally. This was supported by:

"I purposefully began following the superstition because I saw that it positively impacted my performance."(Participant 30).

Analysis suggest that 17 participants believed that they performed their superstitions unintentionally. This was supported by:

"I started following it because my peers and my coaches also used to follow it. When I started using it, I found it to be effective."(Participant 20).

Analysis also suggested that six participants believed that they performed their superstitions both intentionally and unintentionally. This was substantiated by:

"I began following the superstition unintentionally, but then it became a pattern of reinforcement and hence I started performing it intentionally since it improved my performance." (Participant 12)

\section{Basis/ orientation to reality}

Analysis suggest that 18 participants believed that their superstitions hold significant basis in reality and are logical. This was supported by:

"I wear 2 socks in each leg because it improves my grip during the matches." (Participant 1)

Analysis also suggest that 25 participants believed that their superstitions don't hold significant basis in reality and are illogical. This was substantiated by:

"I find it irrational because it just affects our psyche."(Participant 3)

Analysis also suggest that seven participants believed that their superstitions are both logical and illogical, depending on purpose of superstitions. This was supported by:

"I believe that placing, and brand of the kit are illogical, but my rationale behind wearing the socks is logical because it gives me a better grip." (Participant 1)

\section{Evolution/ reduction in superstitions}

Analysis suggested that 13 participants believed that their superstitions have reduced from the time they have started playing their sport. This was supported by:

"Earlier when I began playing, I used to believe a lot in these superstitions. But as I grew up, I saw that teamwork and practise matters the most, than believing in such rituals and practices." (Participant 9)

Analysis also suggest that 16 participants believed that their superstitions have increased from the time they have started using the superstitions. This was supported by:

"We tend to believe that these beliefs won't affect our performance, but as we get connected to the game, we realise that having a faith in such superstitions do impact our performance." (Participant 25)

Analysis also suggest that 21 participants believed that their superstitions have been the same, since the time they have started playing. This was supported by:

"I still follow these superstition, even though I know that there are several other factors which contribute to our performance."(Participant 36)

Looking at the gender differences within different team sports associated with these superstitions, it was analysed that two female players endorsed superstitious beliefs relating to personal appearance which included, tying hair in ponytail (for better concentration), not wearing white shirt (since white can distract), particular wristband. The female football and basketball players mentioned applying kajal before match, wearing grandmother's scarf as a headband (as a belief that grandmother's blessings are with the participant), wearing a black thread (either on hand or foot), using gloves gifted by her coach as a blessing from coach, wearing particular tights and band during matches and having two shoes for matches in order to have a backup option.

The current study also looked at the various superstitions followed by famous sportspersons, which were discussed by the participants. Michael Jordan used to wear his North Carolina college shorts underneath his National Basketball Association shorts in each game throughout his entire career [21]. Top basketball player LeBron James always used to toss moisture-absorbing chalk in the air prior each game. 


\section{DISCUSSION}

The aim of the present study was to qualitatively explore the superstitious beliefs of team sportspersons at intercollegiate level and analyse how these superstitious beliefs affect their sports performance. This study also aimed at analysing the gender differences within different team sports associated with these superstitions. According to the responses received from the participants, the major findings that emerged based on the nature of superstitions included verbal and non-verbal nature. The broader themes which emerged in the verbal nature of superstitions included reciting certain mantras, cheering up as a team, or praying (either silently or loudly as a team). The broader themes which emerged in non-verbal nature superstitions included positioning of kit or equipment related; clothing related and other rituals. Corroborating the results of the current study with the study conducted by Coffin [16], the three kinds of sport superstitions on the basis of their origin were- beliefs and practices which are carried forward from their culture; the long-standing superstitions associated with the sport and personal superstitions which sportspersons assign to their game. The major themes which emerged in the study were also explored by Gregory and Petrie [13] and Becker's [14] study where the nature of superstitions were broadly and logically categorized into equipment, clothing, numbers, practices before and during contests and others (hair, charms, food, coins) and manner of dressing. The findings of the current study were also substantiated by Becker's [14] study that borrowed articles, or new clothing were seen to disrupt an athlete's performance. The significant themes which emerged regarding the origin of such superstitions included family, self and others including a combination of family, self and others. The results of the current study suggested that the majority of superstitions endorsed by the participants were learned through the internal social structures that operate within various sports and that the most important sources in the transmission of such beliefs include family, friends, personal experiences and coaches [17]. The significant findings which emerged regarding the frequency of engaging in such superstitions included during matches or both during matches and practise sessions. According to the study conducted by Burger and Lynn [22] where superstitions of major league baseball players were studied, it was found that their belief in luck having an impact on performance, leaded to an impact on their performance. According to the study conducted by Hobson [23], rituals help in greater immersion in the context of the goal, help in boosting their confidence and motivation that success in goal achievement is likely. According to the study conducted by Hamerman and Morewedge [4], it was found that preference and assignment to a lucky (vs. unlucky) item lead to greater confidence of achieving performance goals but not learning goals. Also, increase in the uncertainity of goal acheievement lead to an increase in superstious behaviours. Another study by Chinchanachokchai [5] asserted that passive superstitious beliefs (low illusion of control) increase the likelihood of engaging in risky behaviors. The major themes which emerged regarding the impact of such superstitions on performance included psychological and reality basis. According to the study conducted by Ofori and others[24], it was suggested that athletes might engage in superstitious behaviour as a means of coping and as a secondary control technique to bring them a sense of control in stressful situations.

The findings of the current study show that the superstitions can be divided into intentional and unintentional nature depending on the purpose served by these superstitious beliefs. According to the study conducted by Blum and others [25], it was found that there is an inherent sense of control associated with superstitious behaviours which is linked with a therapeutic quality in reducing stress. The major themes which emerged regarding the basis which these superstitious beliefs hold in reality include rational, irrational or a combination of both. This corroborated with the findings of the study by Pronin and others [26]; Risen and Gilovich [27] that participants were aware that superstitious thoughts lack rational basis but still tend to engage in them perceiving that it might be beneficial [28-29]. The significant themes which emerged regarding the evolution of these superstitious beliefs included either increase, decrease or constancy in these superstitious beliefs since the time participants started engaging in the sport. This can be corroborated with the results of the study conducted by Rudski and Edwards [30] that the level of perceived expertise in the sport tends to decrease involvement in superstitions (because of increase in the level of control).

Jackson and Baker [31] conducted a case study on an elite rugby kicker and found that the participant utilized varied psychological strategies such as specific mental cues, thought stopping, inverse simulation, visualization and relaxation techniques, which unfortunately differed from attempt to attempt. They 
concluded that the most important causal factor of kicking performance in a competitive environment was the successful application of certain psychological strategies rather than the temporal consistency of the preperformance routine.

Gender differences were also studied in the study and it was concluded that two female players reported endorsing superstitious beliefs relating to personal appearance which included, tying hair in ponytail, not wearing white shirt, particular wristband, applying kajal before match, wearing grandmother's scarf as a headband, wearing a black thread for good luck. These results were substantiated by Gregory and Petrie [13] who analysed women college students where it was reported that women continued to endorse more superstitious beliefs related to personal appearance including uniform, hair, specific hair accessories, team cheers, hands and social pre-game-night activities which stem from general superstitions as compared to men. Whereas the male athletes listed more superstitions relating indirectly to business practices, equipment, religious practices and repetitive actions than did the female athletes. The results of the current study corroborated with the study conducted by Gregory [12], suggesting that team sport athletes provided greater evidence for superstitions relating to equipment and its use; dressing room activities; repetitive rituals; and sports personalities.

But the participants also reported certain superstitions which were practised by individual sportspersons such as wearing charms; lucky jersey numbers; team cheers. According to an Indian study [32], it was analysed that the individual sports are linked to low superstitious beliefs and better decision making level as compared to dual and team sport. Another study conducted [33] asserted that basketball players engaged in supersitions more in terms of Clothing and Appearance, fetish, Team Ritual than Preparation, Game/ Competition, Prayer and Coach.

Corroborating with Samuelsen [15] and Gregory and Petrie's [13] study, evidences of superstitions were found among football, volleyball, and basketball players. The current study also corroborated with Gregory and Petrie [13] study, since the variations in endorsements of types of superstitions relating to uniform, equipment and clothes were most often cited. One of the cricket player mentioned endorsing Sachin Tendulkar's ritual of wearing left batting pad before putting the right one.

Examining the frequency of participants who engaged in superstitious behaviours, it was observed that $78 \%$ participants believed in superstitions. Results of the current study positively corroborated with the study conducted [3], since around $80 \%$ of the population engage in at least one superstitious behaviour before a contest. Another study [20] reported that overall $94 \%$ of the participants reported incidents of superstitions in which $90 \%$ women and $73 \%$ men admitted using superstitions. The most frequently reported SB included number 3 and 13, date 13, and Friday and charms to be unlucky. Women reported SB related to domestic, social affairs, feelings whereas men reported superstitions relating to sports, business related activities. Talking about the origin of these superstitions, most of them reported that they used them because of their family and other elders whereas some attributed them to their teammates who also followed them. This can also be substantiated by another study by Tahir and others [7] that older people play a key role in the prevalence of superstitions.

\section{CONCLUSION}

There is a very high prevalence of depression, anxiety and stress among interns working in Maharashtra which needs to be effectively dealt with on an urgent basis. The strong association of the prevalence of psychological illnesses with many factors and stressors faced by them will help us highlight the contributory factors and suggest strategies to deal with them effectively at a personal, institutional and national level.

Superstitions were widely practised by team sports participants hence the current study concludes that superstitions play a vital role in the lives of sportspersons and have a huge impact on their performance. The various themes which emerged from the data included the nature of the superstitions (verbal and non-verbal) held by the participants; the origin (family, self, combination) of such superstitions; frequency (during matches, both in matches and practise) of engaging in such superstitions; impact of such superstitions on performance (psychological and reality basis); purpose served by these superstitious beliefs (intentional and unintentional nature); basis which these superstitious beliefs hold in reality (rational, irrational or a combination); evolution of such beliefs (increase, decrease or constancy). Also, the gender differences were 
thoroughly studied in the study, concluding that female players reported endorsing superstitious beliefs relating to personal appearance such as tying hair in ponytail, not wearing white shirt, particular wristband, applying kajal before match, wearing grandmother's scarf as a headband, wearing a black thread for good luck.

The future implications of the current study include that in India, majority of people hold certain beliefs, superstitions and values which are likely to affect their performance at various spheres, ranging from their work to family life, to sports, education etc. Since sports is given significant importance in Indian culture, hence while coaching and training sportspersons, coaches should be mindful about them and be sensitive enough to accept these superstitious beliefs. Also, training can incorporate these beliefs for performance enhancement.

The limitations of the current study included limited sample size and dearth of female participants. The current study also didn't study individual sports.

\section{REFERENCES}

1. Maller JB, Lundeen GE. Superstition and emotional maladjustment. J Educ Res 1934;27(8):592-617.

2. Brevers D, Dan B, Noel X, Nils F. Sport Superstition: Mediation of Psychological Tension on NonProfessional Sportsmen's Superstitious Rituals. J Sport Behav 2011;34(1).

3. Schippers MC, Van Lange PA. The Psychological Benefits of Superstitious Rituals in Top Sport: A Study Among Top Sportspersons. J Appl Soc Psychol 2006;36(10):2532-53.

4. Hamerman EJ, Morewedge CK. Reliance on luck: Identifying which achievement goals elicit superstitious behavior. Personal Soc Psychol Bull 2015;41(3):323-35.

5. Chinchanachokchai S, Pusaksrikit T, Pongsakornrungsilp S. Exploring different types of superstitious beliefs in risk-taking behaviors: What we can learn from Thai consumers. Soc Marketing Quart 2017;23(1):47-63.

6. Cherrington J. 'It's just superstition I suppose... I've always done something on game day': The construction of everyday life on a university basketball team. Int Rev Soc Sport 2014;49(5):509-25.

7. Tahir TB, Qureshi SF, Safi T. Superstitions as Behavioral Control in Pakistan. Pak J Soc Sci 2018;38(2).

8. Tahir TB, Qureshi SF, Safi T. Superstitions as Behavioral Control in Pakistan. Pak J Soc Sci 2018;38(2).

9. Neil G, Anderson B, Shappard W. Superstitions among male and female athletes of various levels of involvement. J Sport Behav 1981;4(3):137-41.

10. Wright PB, Erdal KJ. Sport Superstition as a Function of Skill Level and Task Difficulty. J Sport Behav 2008;31(2):100-8.

11. Raalte JL, Brewer BW, Nemeroff CJ, Linder DE. Chance orientation and superstitious behavior on the putting green. J Sport Behav 1991;14(1):41-6.

12. Gregory CJ. Superstitions among male and female intercollegiate athletes and non-athletes of the University of Western Ontario, 1971-1972 (Doctoral dissertation).

13. Gregory C. \& Brian M. Superstitions in Sport. In: Canadian Psycho-Motor Learning and Sports Psychology Symposium. Fourth Annual Conference. 1972 Oct; 23(25): 384-403.

14. Becker J. Superstition in Sports. Int J Sport Psychol 1975;6(3):148-52.

15. Samuelsen R. Superstitions in Sport, The Sports Library of Studebaler -Packard Corp., Detroit, Michigan. 1957.

16. Coffin TP. The Old Ball Game, Herder and Herder Publishers, New York. 1951.

17. Gregory C, Petrie B. Superstitions of Canadian Intercollegiate Athletes: an Inter-Sport Comparison. Int Rev Sport Sociol 1975;10(2): 59-68.

18. Womack M. Why athletes need ritual: A study of magic among professional athletes. Sport Religion 1992;2:191-202.

19. Lonsdale C, Tam JT. On the temporal and behavioural consistency of pre-performance routines: An intraindividual analysis of elite basketball players' free throw shooting accuracy. J Sports Sci 2008;26(3):259-66.

20. Otis LP, Alcock JE. Factors affecting extraordinary belief. J Soc Psychol 1982;118(1):77-85.

21. Damisch L, Stoberock B, Mussweiler T. Keep your fingers crossed! How superstition improves performance. Psychol Sci 20101;21(7):1014-20.

22. Burger JM, Lynn AL. Superstitious behavior among American and Japanese professional baseball players. Basic Appl Soc Psychol 2005;27(1):71-6.

23. Hobson NM, Schroeder J, Risen JL, Xygalatas D, Inzlicht M. The psychology of rituals: An integrative review and process-based framework. Personal Soc Psychol Rev 2018;22(3):260-84. 
24. Ofori PK, Tod D, Lavallee D. An exploratory investigation of superstitious behaviours, coping, control strategies, and personal control in Ghanaian and British student-athletes. Int J Sport Exercise Psychol 2018;16(1):3-19.

25. Blum SH, Blum LH. Do's and dont's: An informal study of some prevailing superstitions. Psychol Rep 1974;35(1):567-71.

26. Pronin E, Wegner DM, McCarthy K, Rodriguez S. Everyday magical powers: The role of apparent mental causation in the overestimation of personal influence. J Personal Soc Psychol 2006;91(2):218-24.

27. Risen JL, Gilovich T. Another look at why people are reluctant to exchange lottery tickets. J Personal Soc Psychol 2007;93(1):12-7.

28. Bashir F, Khanum A, Bashir S. The Role of Female Writers in the Promotion of Popular Literature in Urdu. PUTAJ-Humanities and Social Sciences 2016;23(2):55-61.

29. Killeen PR. Superstition: A matter of bias, not detectability. Science 1978;199(4324):88-90.

30. Rudski JM, Edwards A. Malinowski goes to college: Factors influencing students' use of ritual and superstition. J Gen Psychol 2007;134(4):389-403.

31. Jackson RC, Baker JS. Routines, rituals, and rugby: Case study of a world class goal kicker. Sport Psychologist 2001;15(1):48-65.

32. Bal BS, Singh D, Badwal KK, Dhaliwal GS. Superstitions Behavior and Decision Making in Collegiate Athletes: An Illogical Phenomenon. Adv Phys Educ 2014;4(1):1-6.

33. Deol NS, Singh D. An Analysis of the Components of Superstitions Behavior and Will to Win in Basketball Performance. Am J Psychol 2015;4(5):129-36.

34. Cotterill S. Pre-performance routines in sport: Current understanding and future directions. Int Rev Sport Exercise Psychol 2010;3(2):132-53.

35. Foster DJ, Weigand DA, Baines D. The effect of removing superstitious behavior and introducing a preperformance routine on basketball free-throw performance. J Appl Sport Psychol 2006;18(2):167-71.

36. Gregory CJ. Changes in superstitious beliefs among college women. Psychol Rep 1975;37(3):939-44.

37. Neil GI. Demystifying sport superstition. Int Rev Sport Sociol 1982;17(1):99-124.

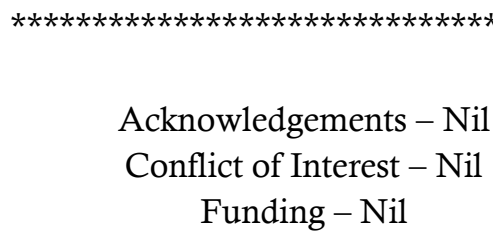

